\title{
Intermediate Stars in Extragalactic Radiosource Fields: Astrometric Measurements
}

\author{
Mirel Birlan \\ Observatoire de Paris-Meudon, DESPA, 5 Place Jules Janssen, 92195 \\ Meudon Cedex, France, e-mail: mbirlan@despa.obspm.fr \\ Gheorghe Bocsa \\ Astronomical Institute of the Romanian Academy, str. Cutitul de \\ Argint-5, 75212 Bucharest 28, Romania, e-mail: gbocsa@roastro.astro.ro
}

\begin{abstract}
The statistical analysis of the $O-C$ and the standard errors for the astrometric RRS2 standard stars, and the analysis of the standard errors for the intermediate PIRS stars for 75 extragalactic radiosource fields are presented. This study was performed at Bucharest Observatory.
\end{abstract}

\section{Introduction}

The astrometric survey at Bucharest Observatory started in 1992 as the framework of the former CONFOR program. CONFOR was an astrometric survey that has been joined by several eastern European institutes. The aim of CONFOR (an acronym for 'Connection of Frames in Optical and Radio regions'), is to establish a link between the fundamental system FK5 and the radio-astronomical coordinate system. For this purpose, the extragalactic catalog of radiosources of Argue and de Vegt (1984) has been considered.

The main concept in the CONFOR has been the use of optical positions of reference stars in the vicinity of extragalactic radiosources to create a reliable basis for a good astrometric reduction of extragalactic radiosources. Gubanov $e t$ al. (1990), and Tel'niuk-Adamchuk et al. (1996) have reported on the CONFOR program.

Two major steps of our program have been considered. These are 1) the astrometric accuracy for the standard and intermediate neighbor stars and 2) the final astrometric measurements for the extragalactic radiosource of each area in the optical domain.

The instrument used to perform the observations necessary for the first step was the Mertz-Prin double astrograph $(\mathrm{f}=6 \mathrm{~m}, \mathrm{D}=38 \mathrm{~cm})$ at Bucharest Observatory. The $2 \times 2$ degree instrument field allowed us to reach stars of $13-14^{\text {th }}$ magnitude on photographic plates, using exposure times between 30 and 50 minutes. Since 1992 a total of 186 extragalactic radiosource fields have been observed with this equipment. In this paper we will present the results concerning 75 of them. 


\section{Observations}

The observations were carried out on the $24 \times 24 \mathrm{~cm}$ astrophotographic plates. Most of them were done at the moments when the stellar fields passed through the meridian. The exposure times were established as a function of the position of the field on the sky: up to 50 minutes for the fields from -20 to 0 degrees in declination, and $30 \mathrm{~min}$ for the fields around 45 degrees declination. The observations were reduced taking into account two intermediate catalogs, RRS2 and PIRS.

The RRS2 catalog contains astrometric positions for 2575 stars in the region from -20 to +90 degrees declination. The average number of RRS2 stars contained in a one square degree area centered on an extragalactic radiosource is 10 , their visual magnitude being less than 10 . The coordinates are reported for mean epoch 1990.5 in the FK5 system, the proper motion also being known. Because the RRS2 stars were observed at several observatories, the rms uncertainities are between $0.010-0.016 \mathrm{sec}$ and $0.12-0.27$ arcsec in right ascension and declination respectively.

The Photographic Intermediate Reference Stars (PIRS) catalogue contains stars from $11^{\text {th }}$ to $14^{\text {th }}$ visual magnitude in a $30 \times 30$ arcminute neighborhood of the radiosources. The astrometric positions of the PIRS stars have been taken from the GSC catalogue. On average, about 25 PIRS stars per each extragalactic radiosource field have been measured. The $30 \times 30$ arcminute areas have been established taking into account the field of the MertzPrin refractor. We consider that a field of $30 \times 30$ arcmin centered in the instrument's field of $2^{\circ} \times 2^{\circ}$ is not affected by aberration.

\section{Results}

Both RRS2 and PIRS measurements were performed with the manually operated ASCORECORD measuring machine. The measurements were reduced using the following catalogues: CAMC, PPM, TYCHO for the RRS2 stars astrometry, and GSC for the PIRS stars. A total of 897 RRS2 stars have been measured in 75 areas. The proper motions in the reference catalogs for the RRS2 stars have allowed us to perform some standard error analyses both in right ascension and in declination, as well as an $(O-C)$ analysis.

As seen in Figures 1 and 2, the $O-C$ analysis shows sharp histograms centered on zero. It is interesting to mention that, depending on the binning chosen for the measurements, the $(O-C)_{\delta}$ values are centered on zero. Nevertheless, a slight $(O-C)_{\delta}$ shift through negative values (Figure 2) can be easily seen for large binning. This fact could not be explained.

A total of 3928 PIRS stars have been also measured for the 75 areas. For the PIRS stars, since the GSC catalog does not include proper motions, only the individual standard errors could be computed. As seen in Figures 3 and 4, the larger part of the PIRS stars have residuals smaller than 1 arcsecond. The large residuals (larger than 2.5 arcseconds) are probably due to pointing error. 


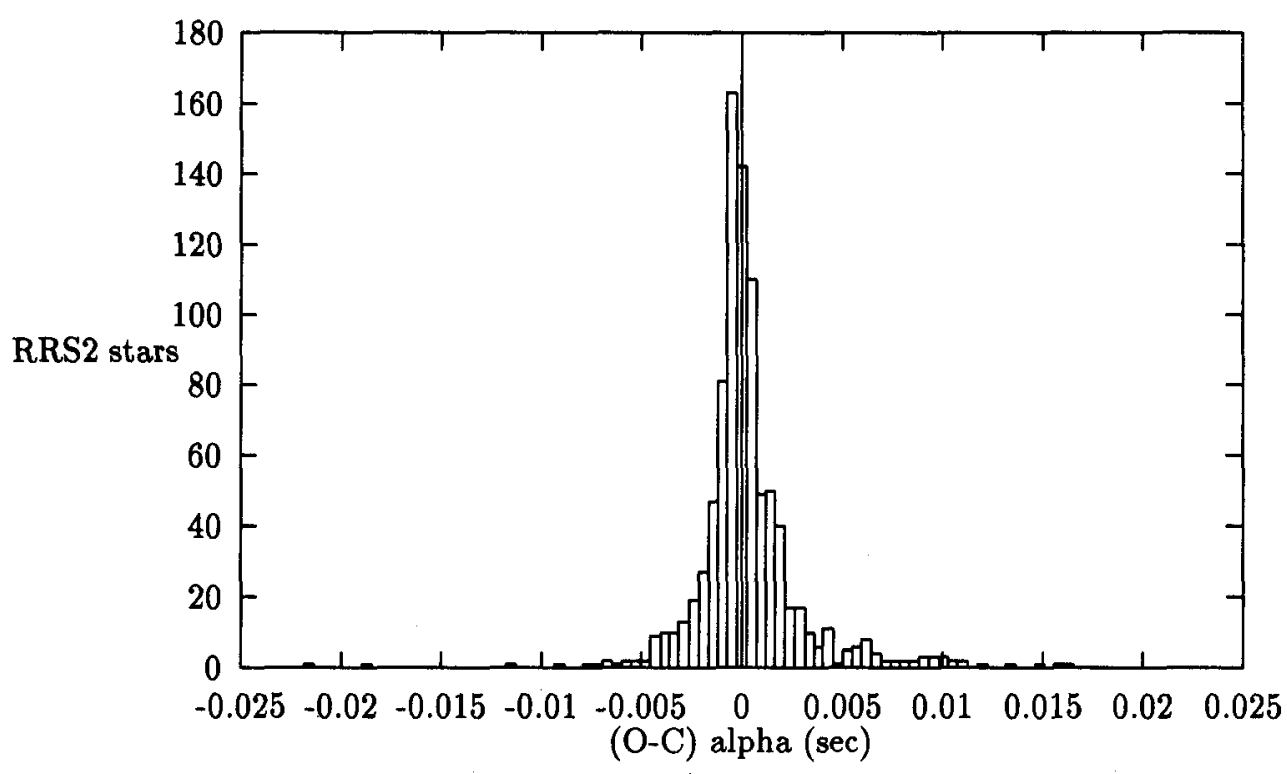

Figure 1. $O-C$ in right ascension for the RRS2 stars measured in the 75 extragalactic radiosource areas.

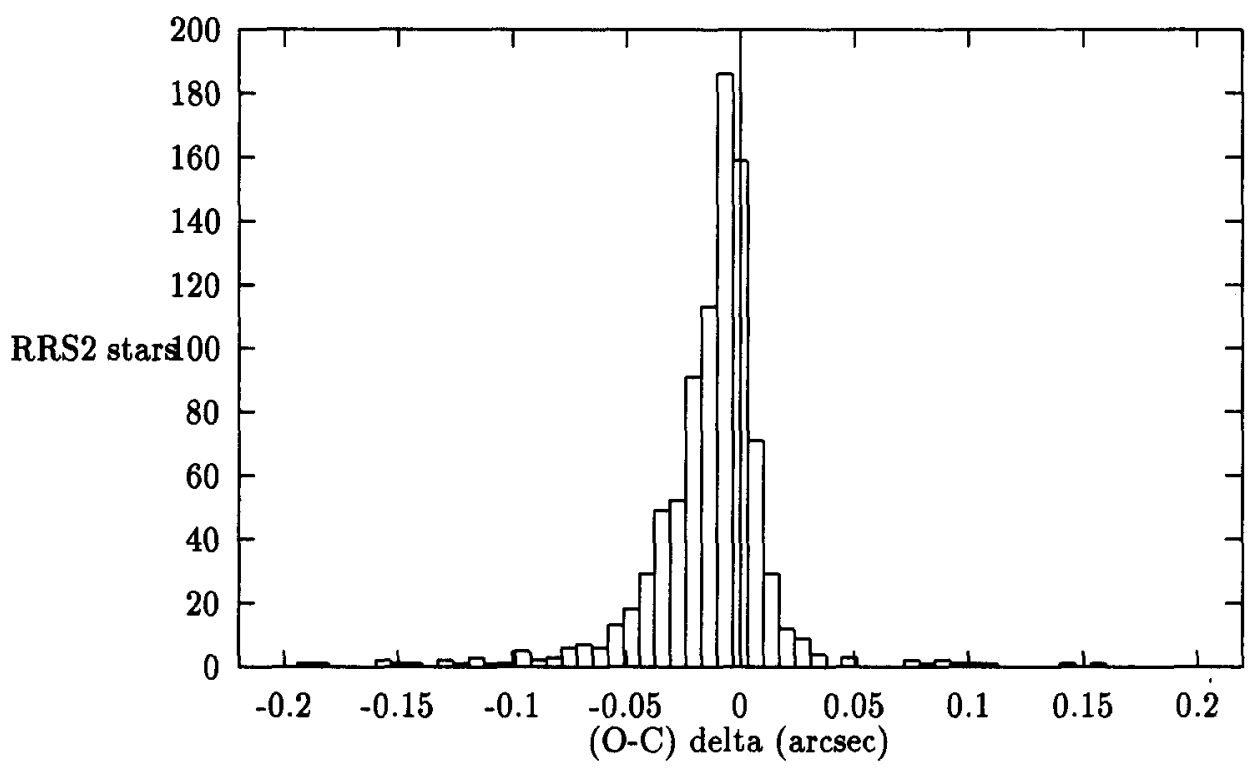

Figure 2. $O-C$ in declination for the RRS2 stars measured in the 75 extragalactic radiosource areas. 


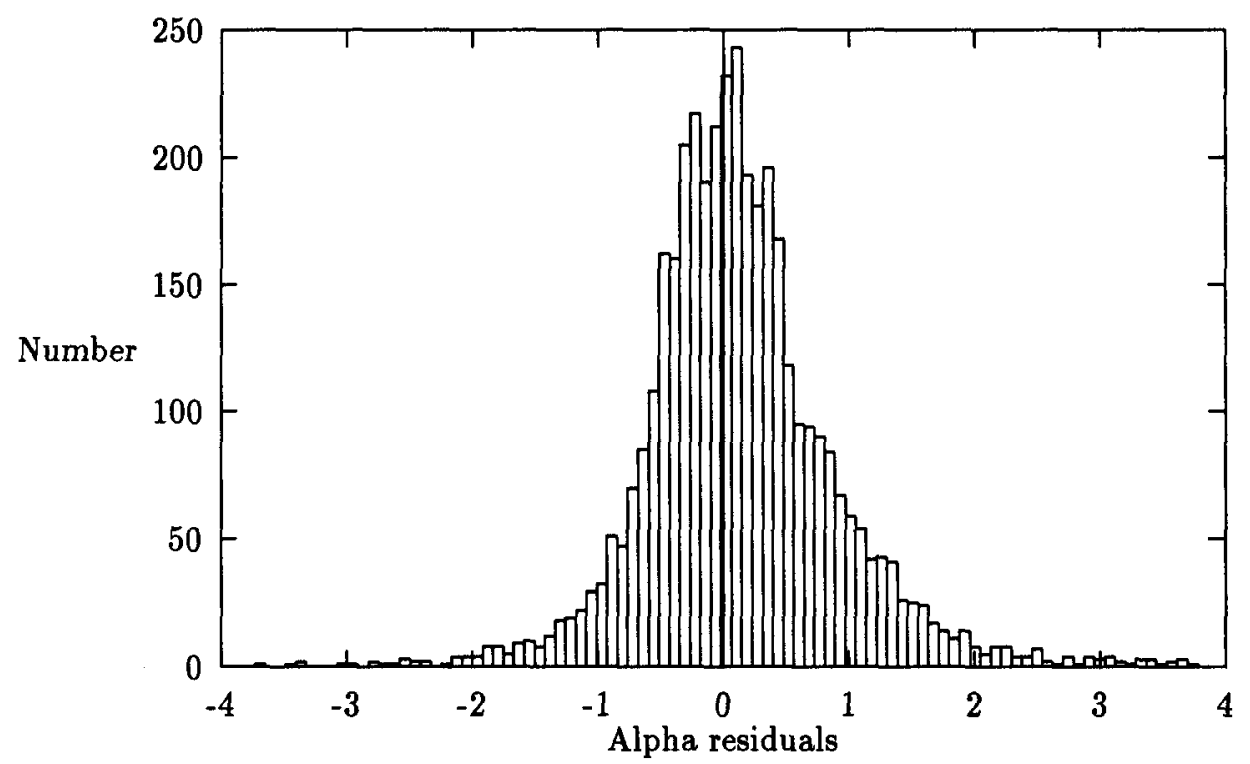

Figure 3. Residual histogram in right ascension for the PIRS stars measured in the 75 extragalactic radiosource areas. Units are arcseconds.

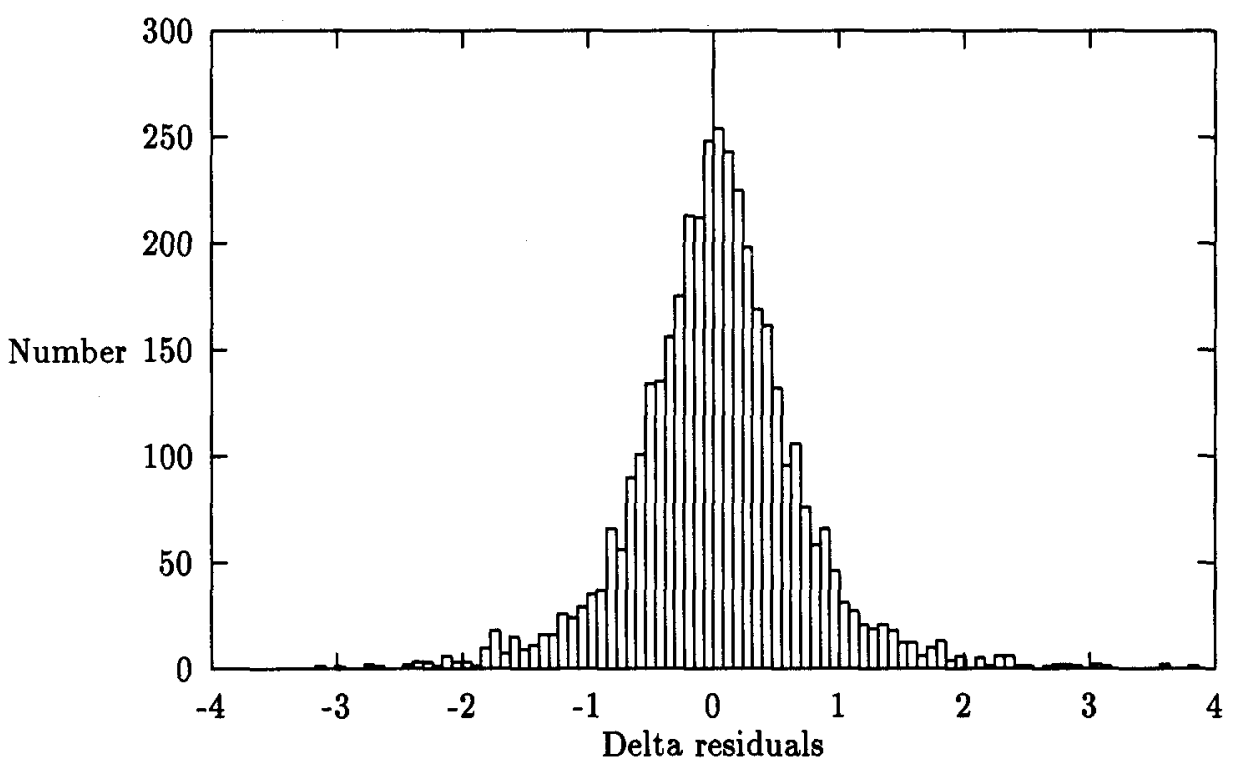

Figure 4. Residual histogram in declination for the PIRS stars measured in the 75 extragalactic radiosource areas. Units are arcseconds. 


\section{Conclusions}

The final product of our astrometric survey will be an astrometric catalog giving the precise photographic astrometry for the PIRS stars. Since the proper motion of the PIRS stars are unknown, it will be impossible to compute the $(O-C)$ s. A future project will be devoted to reductions using TYCHO stars. However, our measurements reveal distributions that could very well fit the two Gaussians centered on zero. The full half widths of these Gaussian functions are 1.15 arcseconds and 1.04 arcseconds for residuals in right ascension and declination respectively.

\section{References}

Argue, A.N. and de Vegt, C., 1984, Astron. Astrophys., 130, 191.

Bocsa, G., 1999, Romanian Astronomical J. (in press).

Gubanov, V.S., Kumkova, I.I., Tel'nyuk-Adamchuk, V.V., 1990, in Inertial coordinate system on the sky reference frames. Proceeedings of IAU Symposium 141, Kluwer Publ., Dordrecht.

Tel'niuk Adamchuk, V., Molotaj, O., Bocsa, G., Pinigin, G., Shulha, O., Gorel, L., Volyanska, M., 1996, in IV International Workshop on Positional Astronomy and Celestial Mechanics, Lopez Garcia, A., Yagudin, L.I., Martinez Uso, M.J., Barbero A.C., Yagudina, E.I., Morano, J.A. (eds.), Valencia 1998 . 